\title{
Eco-technologies for retrofitting social housing stock: strategies for an Italian case study
}

\author{
A. Boeri \& D. Longo \\ University of Bologna, DAPT, Faculty of Architecture of Cesena, Italy
}

\begin{abstract}
While there are an increasing number of new projects aiming at combining high residential quality and low power consumption, it is clear that the main challenges in the short term concern the performance upgrading of the existing residential building stock.

As a matter of fact, when compared to new buildings construction methods, this kind of intervention reduces the consumption of land and energy and could be applied to a large portion of buildings characterized by low architectural quality and low performances. Social housing stock consumes high energy and does not offer suitable levels of comfort. This type of building is characterized by different variables, ranging from its historical background to the more recent suburban additions, and requires flexible methods of approach to define objectives and modalities of intervention.

In Italy, the condition of the existing residential stock is not very satisfactory. Therefore, it is important to define methods of requalification to improve energy and morphological performances of construction.

This paper describes the status of some of the Italian high density suburban areas, focusing in particular on a district in Bologna in the Emilia Romagna region (IT).

This paper aims at identifying intervention strategies for morphological and energy improvement. Their effectiveness in terms of reducing the energy requirements will be verified with a simulation model of the behaviour of one of the building subject of retrofitting.

Keywords: social housing, sustainability, energy saving, retrofitting.
\end{abstract}




\section{Introduction}

The increasing awareness of the importance of the environmental issues has brought the need of reducing energy consumption in the public eye and consequently also the one of the social housing stock, which, similarly to the existing private residential stock (figs. 1-2), has poor energy performance mainly due to the large heat losses through its envelope.
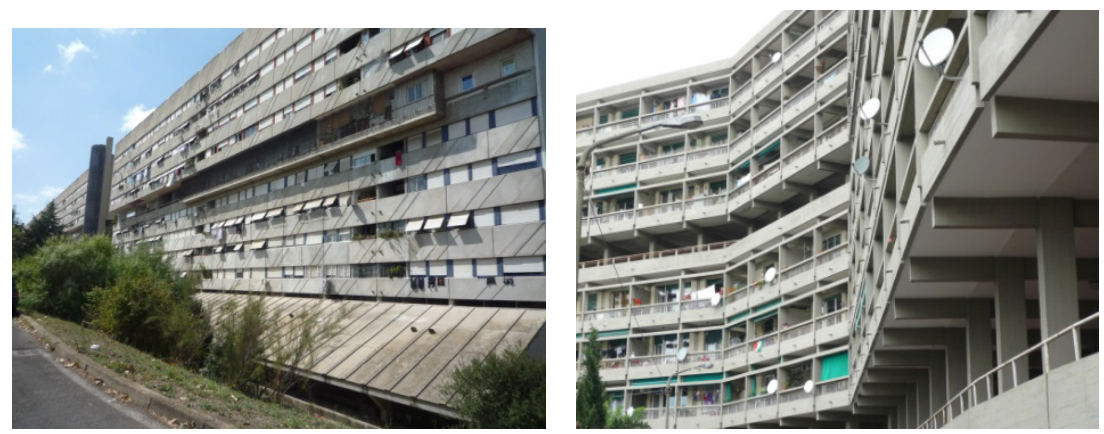

Figures 1-2: $\quad$ Social housing stocks in Italy: Corviale in Rome and Forte Quezzi in Genoa.

About 400.000 units were built before 1990 and therefore without any thermal insulation. They also have obsolete systems and unsuitable windows and door frames. In addition there are problems in terms of not sufficient ventilation or lighting, humidity, usage of materials later found to be hazardous. But then, buildings erected starting from the period immediately after the Second World War, and in particular, social housing buildings were produced with limited budgets since they were built for low-income families, even if supported by public funds. Furthermore, this type of housing stock was created to meet the situation of real emergency the Country was in, and often in these cases quantity takes over quality [1].

\section{The housing heritage in Italy}

Italian housing stock is characterized by low performances, as it consumes a high level of energy and does not offer suitable levels of comfort. The level of energy demand for winter heating, the common benchmark, is extremely high: for older buildings; it is around $250 \mathrm{kWh} / \mathrm{m}^{2} \mathrm{y}$, decreasing to an average of about $150 \mathrm{kWh} / \mathrm{m}^{2} \mathrm{y}$ in constructions built after the coming into force of Law n.10 of 1991. The comparison of these buildings performance with the more energyefficient ones is rather discomforting: a passive building does not consume more than $15 \mathrm{kWh} / \mathrm{m}^{2} \mathrm{y}$; a Gold CasaClima building consumes no more than $10 \mathrm{kWh} / \mathrm{m}^{2} \mathrm{y}$, a Class A building in the Emilia-Romagna region consumes less than $25 \mathrm{kWh} / \mathrm{m}^{2} \mathrm{y}$; production of hot water included. 
Therefore, it is important to define flexible methods of requalification to improve energy and morphological performances of construction in relation to the different operational and climatic areas $[2,3]$.

\section{The case study}

Within the Programme of Scientific Research of National Interest (PRIN), in 2010 the Italian Ministry for Education University and Research has supported the Research "Renovation, regeneration and valorisation of social housing settlements built in the suburban areas in the second half of last century", conducted by a team of researchers from different Italian Universities.

In this context, the Research Unit of the University of Bologna was entrusted with a case study, that of the Pilastro neighbourhood, an important settlement of public housing located in the Emilia-Romagna regional capital, Bologna.

Pilastro is a typical high-density settlement, with large housing estates that have suffered, since their creation, conditions of physical marginalization, which eventually led to issues related to the social aspect.

Pilastro was developed over a period of time starting from the end of the Fifties, when the first plan was designed, up to the mid-Eighties. It is placed at the centre of an urban area that the city of Bologna considers strategic in terms of future expansion and where it is carrying out major interventions, including the construction of infrastructures.

Today, Pilastro, with its 7,500 residents, has a provision of neighborhood facilities and has solved most of its problems of social and public nature, however the strong spirit of participation and cohesion amongst the residents is no longer present. Streets are empty, squares do not function as meeting and socializing places and the few businesses on the premises are now empty.

The strong monotonicity of fronts (see fig. 3), which in the extreme case of the "Virgolone" building doesn't help to create an "urban effect" also consists in the sense of recognition and belonging. The poor quality of its external finishes and the degradation, so evident in many buildings, reveal the current difficulties

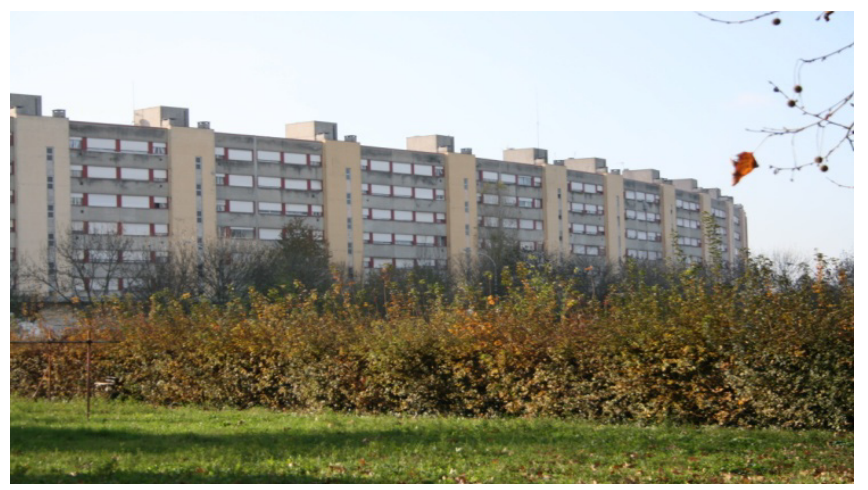

Figure 3: The Virgolone building in the Pilastro neighborhood. 
of ACER, the managing body of the public housing stock in Bologna, in carrying out any maintenance work or any other work these buildings need.

Currently, the housing estate interior, carefully designed and articulated in various types and sizes at the time, shows limitations to its usability and features a distribution that often makes them unsuitable to the current needs. The buildings constructed during the first period (1966-1975) have a traditional structure made of reinforced concrete beams and pillars, which makes them more flexible and adaptable for a possible retrofitting work, when compared with the more recent built Virgolone building and the Towers.

In fact, they are made with a tunnel system, consisting of a reinforced concrete structure with baffles that are repeated and positioned at a maximum distance of 3.80 meters, and with a prefabricated system, respectively.

Another severe weakness of the built heritage of Pilastro is its high energy needs.

\section{Energy analysis of the reference building}

The energy evaluation of Virgolone was carried out with a dual purpose: providing an in-depth detailed report of the current situation and identifying those elements of critical importance in order to define the strategies of retrofitting considered most effective in terms of costs and benefits.

The building energy need evaluation was carried out through the definition of a simulation model that took into consideration the geometric data of the building, its envelope components and its plants system.

To simplify the simulation operation, once considered the characteristics of Virgolone, it was decided to set the analytical calculation on a single portion of the building, and then extend the final result to the entire construction.

This evaluation method was specifically defined for the case study of reference as an alternative to an analytical evaluation of each single unit. The reason for this choice lies in the repetitive nature and large size of the building, that house 552 residential units, whose accurate assessment would have involved high costs and spending significant time, not compatible with the ones related to our research. The results obtained through the application of this method of assessment were validated when compared to the values of the actual consumption reported during the three-year period 2007-2009.

The building presents a modular aggregation developing along a curve axis, with a variable exposure of the main facades. The simulation model was set by taking as reference the portion of building corresponding to substation n. 18 of the district heating network, with facades facing north and south and consisting of 5 stairwells servicing 75 units of different size.

Inside the compact shape of Virgolone, developing on eight over-ground floors and one underground, the heated volume includes the seven upper floors and this portion is limited vertically by the north and south facade, horizontally by the roof and at the bottom by the floor that covers the gallery on the ground floor. 
Table 2: $\quad$ CVE2_Outer vertical closure 2-Thermal physical specifications.

\begin{tabular}{|c|c|c|c|}
\hline Thermal transmittance $\mathrm{U}\left[\mathrm{W} / \mathrm{m}^{2} \mathrm{~K}\right]$ & 0,97 & \multirow{2}{*}{\multicolumn{2}{|c|}{$-\bar{T} \pi^{-}$}} \\
\hline Total thickness [cm] & 20 & & \\
\hline Dumping factor Fd [\%] & $57,31 \%$ & \multirow{3}{*}{ I } & \multirow{3}{*}{$\mathrm{E}$} \\
\hline Time lag $\varphi[\mathrm{h}]$ & $5 \mathrm{~h} 35^{\prime}$ & & \\
\hline Periodical thermal transmittance $\mathrm{Y}\left[\mathrm{W} / \mathrm{m}^{2} \mathrm{~K}\right]$ & 0,553 & & \\
\hline
\end{tabular}

Table 3: $\quad$ CVE3_Outer vertical closure 3 - Thermal physical specifications.

\begin{tabular}{|l|l|l|}
\hline Thermal transmittance U $\left[\mathrm{W} / \mathrm{m}^{2} \mathrm{~K}\right.$ & 1,11 & \multirow{3}{*}{} \\
\hline Total thickness $[\mathrm{cm}]$ & 21 & \\
\hline Dumping factor Fd [\%] & $24,55 \%$ & \\
\hline Time lag $\varphi[\mathrm{h}]$ & $6 \mathrm{~h} 42$ & \\
\hline Periodical thermal transmittance $\mathrm{Y}\left[\mathrm{W} / \mathrm{m}^{2} \mathrm{~K}\right]$ & 0,273 & \\
& & \\
\hline
\end{tabular}

Table 4: COE1 Outer horizontal closure $1-$ Thermal physical specifications.

\begin{tabular}{|l|l|l|}
\hline Thermal transmittance $\mathrm{U}\left[\mathrm{W} / \mathrm{m}^{2} \mathrm{~K}\right]$ & 1,12 \\
\hline Total thickness [cm] & 32 \\
\hline Dumping factor Fd [\%] & $49,97 \%$ \\
\hline Time lag $\varphi[\mathrm{h}]$ & $5 \mathrm{~h} 38$ \\
\hline Periodical thermal transmittance $\mathrm{Y}\left[\mathrm{W} / \mathrm{m}^{2} \mathrm{~K}\right]$ & 0,558 & \\
\hline
\end{tabular}

Table 5: CS_Superior closure/roof - Thermal physical specifications.

\begin{tabular}{|l|l|l|l|}
\hline Thermal transmittance $\mathrm{U}\left[\mathrm{W} / \mathrm{m}^{2} \mathrm{~K}\right]$ & 0,62 & \\
\hline Total thickness $[\mathrm{cm}]$ & 27 & \\
\hline Dumping factor $\mathrm{Fd}[\%]$ & $21,05 \%$ & \\
\hline Time lag $\varphi[\mathrm{h}]$ & $8 \mathrm{~h} 23$ & & \\
\hline Periodical thermal transmittance $\mathrm{Y}\left[\mathrm{W} / \mathrm{m}^{2} \mathrm{~K}\right]$ & 0,131 & & \\
& & & \\
\hline
\end{tabular}

Glazed element:

Steel window frame with a single pane $\mathrm{U}=5,76 \mathrm{~W} / \mathrm{m}^{2} \mathrm{~K}$

As regards the systems data, the district heating system meeting the winter needs of the entire neighborhood was considered.

The need for thermal energy necessary for the production of hot water, independently produced in each unit, was calculated by the software as equal to $23.50 \mathrm{kWh} / \mathrm{m}^{2} \mathrm{y}$. Once the data related to the systems were entered and the types of thermal bridges were analytically defined, the PE indexes per housing typologies were deduced. 
The final result is a PEtot index value equal to $118.54 \mathrm{kWh} / \mathrm{m}^{2} \mathrm{y}$.

The PEtot index value allowed to classify the building in energy class D (fig. 5), according to DLA156/2008 and subsequent amendments, in force in Emilia Romagna.

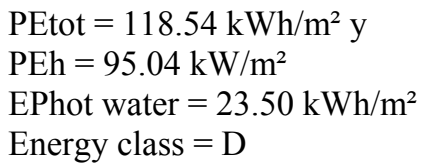

PEtot $=118.54 \mathrm{kWh} / \mathrm{m}^{2} \mathrm{y}$

EPhot water $=23.50 \mathrm{kWh} / \mathrm{m}^{2}$

Energy class $=\mathrm{D}$

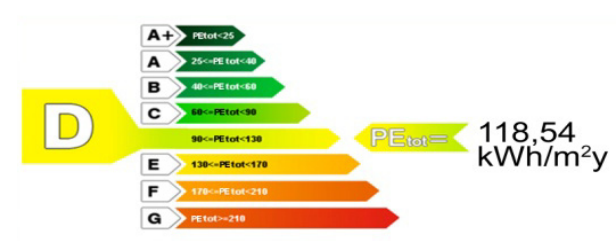

Figure 5: $\quad$ Final results of current status analysis.

\subsection{Analysis of the energy behavior and hypothesis of intervention on the reference building: Virgolone}

The energy audit of the current status highlighted the reason why the reference building, Virgolone, presents very high energy needs: they are mainly due to the construction methods used, as well as to the techniques and materials used. Furthermore the evident degree of decay this building is in also causes a significant reduction in its energy performance and a further worsening of the levels of comfort.

With the aim to improve the level of energy efficiency, some interventions for an energy retrofitting of the building and its envelope were defined (fig. 6), taking into consideration their impact in terms of performance and financial resources.

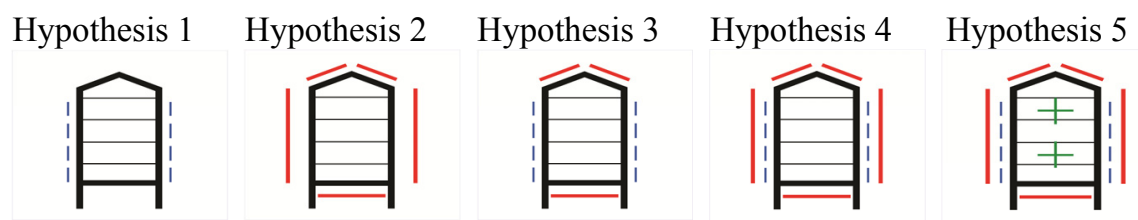

Figure 6: Schemes of different hypothesis of intervention.

These scenarios may be considered as a sequence of different stages of intervention that may be carried out at different times, depending on the financial resources available: the strategies of intervention identified were defined so to be able to apply them individually or in an integrated way, enabling to achieve increasingly higher levels of quality.

\subsubsection{Hypothesis 1: replacement of windows (values by law)}

The first scenario of intervention consists in a replacement of the existing doors and windows with new ones made of wood, provided with air chamber. This replacement intervention is of fundamental importance for bringing them up to standard in compliance with the current regulations on the matter of energy 
efficiency. Virgolone presents windows mainly made of aluminum, with profiles without thermal break and single glazed. The total thermal transmittance of these windows can be assessed as equal to $5.76 \mathrm{~W} / \mathrm{m}^{2} \mathrm{~K}$, a value significantly higher than the one set as top limit by law at $2.2 \mathrm{~W} / \mathrm{m}^{2} \mathrm{~K}$, required for buildings in climatic area $\mathrm{E}$.

The glazed elements form a significant portion of the vertical envelope and are the main causes of heat loss. As highlighted by the results of the blower door test carried out, the windows have a very low degree of air-tightness capacity, therefore they are not capable of meeting the minimum level of acoustic and thermo-hygrometric comfort requirement.

The application of wooden framed windows, provided with profiles of $70 \mathrm{~mm}$, double glazing (4-12-4) and air cavity, with a low transmittance treatment on the inside surface, is a possible solution. The overall thermal transmittance is of $2.1 \mathrm{~W} / \mathrm{m}^{2} \mathrm{~K}$, in accordance with the limits set by the relevant standard.

Compared to the current status, the value of PEtot index changes from the current 118.54 to $90.20 \mathrm{kWh} / \mathrm{m}^{2} \mathrm{y}$ (fig. 7), including the primary energy need for the production of hot water, equal to $23.50 \mathrm{kWh} / \mathrm{m}^{2} \mathrm{y}$, and primary energy need for winter heating equal to $66.70 \mathrm{kWh} / \mathrm{m}^{2} \mathrm{y}$. Therefore, just with a simple replacement of the clear elements it is possible to save $28.34 \mathrm{kWh} / \mathrm{m}^{2} \mathrm{y}$ equal to $31.4 \%$ of the current energy needs.

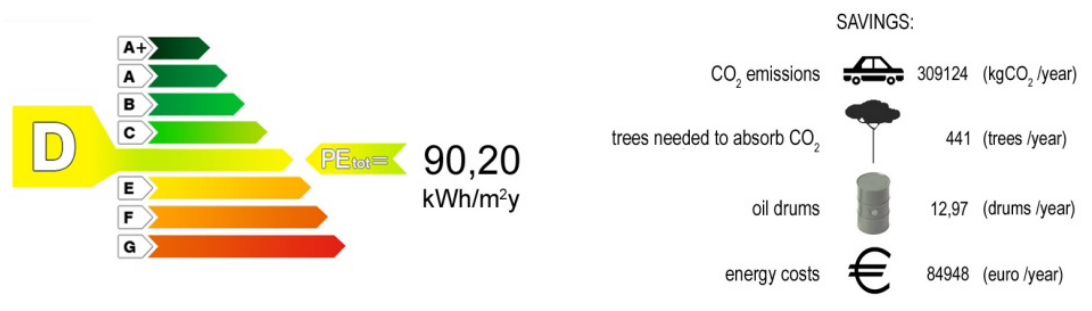

Figure 7: $\quad$ Final results of hypothesis 1.

\subsubsection{Hypothesis 2: thermal insulation of the envelope (value by standard)}

The second scenario foresees interventions on the opaque building envelope in order to reduce the thermal transmittance. The external vertical closures currently consist of: concrete panels with a thickness of $15 \mathrm{~cm}$ plastered on their external surface, provided with a layer of inside insulation of foamed polystyrene panels $3 \mathrm{~cm}$ thick and finished with a plasterboard sheet facing inside. These walls have an overall thickness of $21 \mathrm{~cm}$ and a thermal transmittance of $\mathrm{U}=1.11 \mathrm{~W} / \mathrm{m}^{2} \mathrm{~K}$, substantially higher than the ones set by law.

The upper roof floor is made of a concrete bearing slab $15 \mathrm{~cm}$ thick and a plate covering roofing. The overall thickness of the roof is about $27 \mathrm{~cm}$, with a thermal transmittance of $0.62 \mathrm{~W} / \mathrm{m}^{2} \mathrm{~K}$.

The lower closure of the heated volume consists of a floor that divides the units on the first floor from the ground floor gallery. This horizontal closure consists of a flat concrete slab $15 \mathrm{~cm}$ thick with an air layer of $12 \mathrm{~cm}$, a glass wool insulation layer $2 \mathrm{~cm}$ thick and false ceiling of cement, with a suspected 
presence of asbestos, whose removal and disposal has been forecasted. The overall thickness of this closure is of $32 \mathrm{~cm}$ with a high value of thermal transmittance equal to $\mathrm{U}=1.12 \mathrm{~W} / \mathrm{m}^{2} \mathrm{~K}$.

Despite the fact that the opaque vertical closures are in fair conditions in terms of materials used for their construction, to improve the building energy efficiency, it is necessary to carry out an intervention consisting of the application of semi-rigid panels of medium density mineral wool, $8 \mathrm{~cm}$ thick, on the outer surface so to reduce their transmittance to $0.33 \mathrm{~W} / \mathrm{m}^{2} \mathrm{~K}$.

On the roof, a cloth, functioning as moister break, is added to the concrete slab. On this cloth, a layer of medium density rock wool panels $6 \mathrm{~cm}$ thick, is positioned so to reduce transmittance to $0.29 \mathrm{~W} / \mathrm{m}^{2} \mathrm{~K}$.

On the ground floor soffit, it is possible to apply a layer of $10 \mathrm{~cm}$ of medium density rock wool and a sheet of cement as finishing. This stratigraphy ensures a transmittance of $0.30 \mathrm{~W} / \mathrm{m}^{2} \mathrm{~K}$, corresponding to the minimum standard set by the regulations for buildings located in area $\mathrm{E}$.

This intervention allows to obtain a PEtot index equal to $85.71 \mathrm{kWh} / \mathrm{m}^{2} \mathrm{y}$ (fig. 8), corresponding to energy class $\mathrm{C}$ for the Emilia Romagna region.

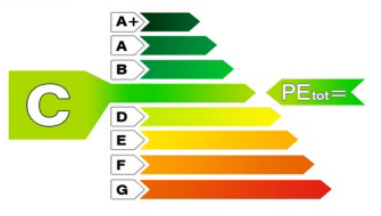

85,71

$\mathrm{kWh} / \mathrm{m}^{2} \mathrm{y}$

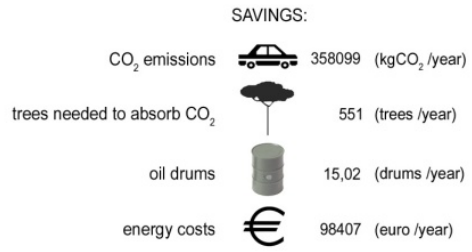

Figure 8: $\quad$ Final results of hypothesis 2.

\subsubsection{Hypothesis 3: replacement of windows, thermal insulation of opaque horizontal closures (value by standard) + installation of solar panels for hot water production}

The third scenario of intervention presumes a partial application of the measures described in the first two scenarios above plus the installation of solar energy panels.

On the basis of the current energy status and cost/benefit critical analysis carried out during the research, this stage consists of an insulation of the horizontal opaque closures (lower and upper) only and not of the vertical ones, under the assumption that a windows replacement is necessary.

This interventions on the envelope offer a solution to the two main problems related to the heat losses, identified in the glazed surfaces of the units positioned on the intermediate levels, and in the horizontal closures of flats on the first floor (lower floor) and on the seventh floor (roof), respectively.

In addition, this strategy foresees the installation of solar panels, in order to produce hot water. This installation would help to significantly reduce energy consumption by using a free source of solar energy; it is a non-invasive intervention, since it is installed on the roof without having to make changes to the indoor spaces. This allows US to reduce the amount of primary energy need for $\mathrm{HW}$, changing the relevant PE from 23.50 to $8.90 \mathrm{kWh} / \mathrm{m}^{2} \mathrm{y}$. 
The combined effect of these measures would reduce the total energy need (PEtot) to $61.49 \mathrm{kWh} / \mathrm{m}^{2} \mathrm{y}$ (fig. 9); a value allowing this building to reach an energy class C, in accordance with DAL 156/2008 and subsequent amendments, currently in force in the Emilia Romagna region.

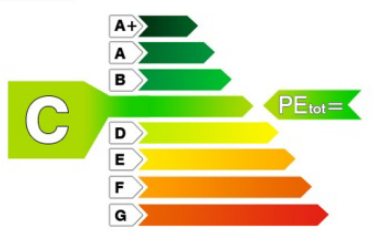

61,49

$\mathrm{kWh} / \mathrm{m}^{2} \mathrm{y}$

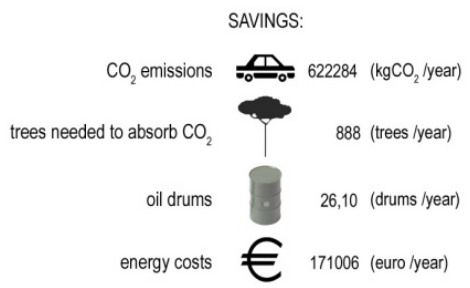

Figure 9: $\quad$ Final results of hypothesis 3.

\subsubsection{Hypothesis 4: replacement of the windows, thermal insulation of the entire opaque envelope (value by standard) + installation of solar panels for hot water production}

The fourth scenario consists in a total integrated application of all measures provided in the first two scenarios. It involves the replacement of the windows with new ones of higher performances, and an addition of a layer of thermal insulation on all the opaque portions of the envelope, so that all closures - either opaque or clear- reach the minimum values of transmittance set by law.

As in the previous scenario, the installation of solar panels for the production of hot water is also foreseen, since they reduce fuel consumption and bring about benefits of environmental nature, thanks to a reduction of $\mathrm{CO}_{2}$ emissions released into the atmosphere.

By applying this strategy of retrofitting, the building limits its primary energy need to $44.09 \mathrm{kWh} / \mathrm{m}^{2} \mathrm{y}$ (fig. 10), including both the need for the production of hot water, equal to $8.90 \mathrm{kWh} / \mathrm{m}^{2} \mathrm{y}$; and the one for winter heating, equal to $35.19 \mathrm{kWh} / \mathrm{m}^{2} \mathrm{y}$. This intervention allows the building to reach the energy class B, according to DAL 156/2008 and subsequent amendments, currently in force in the Emilia Romagna region.
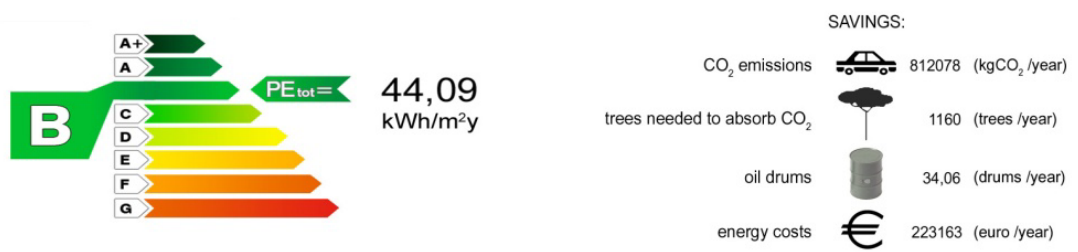

Figure 10: $\quad$ Final results of hypothesis 4.

\subsubsection{Hypothesis 5: replacement of windows, thermal insulation (best values) + installation of solar panels for the production of hot water}

This fifth scenario of intervention is similar to the previous one, the only difference lies in the fact that, in this case, solutions with a higher level of performance are foreseen, so to achieve a result of significant improvement of 
the current status. On the vertical closures the application of $12 \mathrm{~cm}$ thick, semirigid medium density mineral wool panels is provided as external insulation, so to ensure a thermal transmittance of $0.22 \mathrm{~W} / \mathrm{m}^{2} \mathrm{~K}$ and a thermal lag equal to 8 hours and 50 minutes.

The thermal insulation of the roof is improved by applying a layer of rock wool panels $16 \mathrm{~cm}$ thick on the existing concrete panels, allowing us to obtain a transmittance of $0.16 \mathrm{~W} / \mathrm{m}^{2} \mathrm{~K}$ and a thermal lag equal to $13 \mathrm{~h} 49$ '.

On the soffit of the first floor, medium density rock wool panels, $16 \mathrm{~cm}$ thick, and a fiber-cement false ceiling are applied, allowing for an airspace of $3 \mathrm{~cm}$ between the insulation layer and the false ceiling, capable of increasing the insulation performance of this closure. This lower slab reaches a thermal transmittance of $0.2 \mathrm{~W} / \mathrm{m}^{2} \mathrm{~K}$ and generate a thermal lag of $8 \mathrm{~h} 57$ '.

The current window frames of aluminum could be replaced with wooden ones, providing a thermal transmittance equal to $\mathrm{Uw}=1.1 \mathrm{~W} / \mathrm{m}^{2} \mathrm{~K}$, with double glazing (4-16-4) and a cavity saturated with argon gas $\left(\mathrm{Ug}=0.9 \mathrm{~W} / \mathrm{m}^{2} \mathrm{~K}\right)$.

Lastly, as in the previous two hypothesis, the installation of solar panels for the production of hot water is foreseen.

This retrofitting scenario guarantees a building's primary energy need of $24.86 \mathrm{kWh} / \mathrm{m}^{2} \mathrm{y}$ (fig. 11), including the primary energy need for hot water, equal to $8.90 \mathrm{kWh} / \mathrm{m}^{2} \mathrm{y}$, and the primary energy demand for winter heating, equal to $16.96 \mathrm{kWh} / \mathrm{m}^{2} \mathrm{y}$.

This standard corresponds to the energy class A +, according to DAL $156 / 2008$ and subsequent amendments, in force in the Emilia Romagna region.
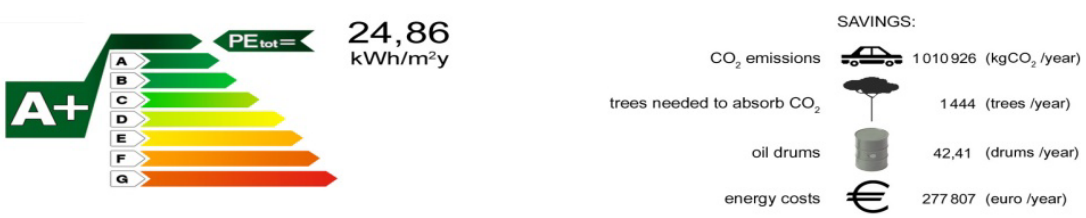

Figure 11: Final results of hypothesis 5.

\section{Financial analysis and evaluations}

The financial analysis was conducted using the discounted cash flow approach, in order to compare the five different scenarios [4]. The pay-back period has been used to verify the amount of time needed to recover the initial investment, considering the inflows and the outflows.

The cost-effectiveness of interventions is consequently calculated adding up the savings that every solution can generate after taking into account the time values of money.

So, the economic analysis takes into consideration the initial cost and energy costs of each intervention and the energy savings obtained in the long period.

For each hypothesis of intervention the savings generated during the 30 years period and the related pay-back period are reported (Tab. 6). In the same table, 
Table 6: $\quad$ Pay-back time, Net Present Values and savings generated by each solution.

\begin{tabular}{lccr}
\hline Interventions & $\begin{array}{c}\text { Pay-back } \\
\text { period }\end{array}$ & NPV & $\begin{array}{r}\text { Savings } \\
\text { (project) }\end{array}$ \\
\hline Hypothesis n.1 & 17 & 814.261 & 2.056 .340 \\
\hline Hypothesis $\mathrm{n} .2$ & 27 & 220.485 & 2.375 .791 \\
\hline Hypothesis n.3 & 19 & 1.285 .861 & 4.099 .008 \\
\hline Hypothesis n.4 & 21 & 1.330 .711 & 5.337 .002 \\
\hline Hypothesis $\mathrm{n} .5$ & 21 & 1.843 .644 & 6.634 .050 \\
\hline
\end{tabular}

the different Net Present Values (NPV) of discounted costs and savings are reported.

\section{Conclusions}

As regards energy retrofitting interventions, it is not possible to identify the best strategy ever: the most convenient solution may be considered such only when it contemplates the needs to meet and the priorities, in terms of technological, financial, energy or functional characteristics, of each specific case.

The retrofitting of the "Virgolone" building project was articulated in a series of steps of interventions aiming at achieving several goals of quality, in relation to the assigned priorities.

The mark of higher performance that can be achieved in terms of energy efficiency does not automatically result in the best option in terms of financial costs, which in reality is the crucial parameter when selecting intervention strategies.

For instance, the solution corresponding to the fifth and last hypothesized scenario for Virgolone, capable of generating the greatest benefits in the long term, from an energy and environmental point of view, is also the one that requires the greatest initial financial effort.

A financial evaluation of the various scenarios of interventions, interrelated with the energy performance results, is fundamental for identifying the best costs/performance ratio. This interrelation allows us to select, amongst all possible scenarios, the ones capable of maximizing the effectiveness of the investment, that is to say the solutions from which it is possible to obtain maximum results with minimum costs.

\section{References}

[1] AA., Una nuova stagione per l'housing, Be-Ma, Milano, 2009.

[2] Malighetti L. E., Recupero edilizio e sostenibilità, Il sole 24 ore, Milano, 2004.

[3] Di Giulio R. (edited by), Suburbanscapes, Alinea, Firenze, 2012.

[4] Brown G., Matysiak G., Real estate investment, a capital market approach, FT Prentice Hall London, 2000. 